\title{
HERRAMIENTAS PARA LA EVALUACIÓN DEL BULLYING
}

\author{
JOSÉ MARÍA AVILÉS MARTÍNEZ
}

\section{RESUMEN}

El bullying es un fenómeno que ha despertado interés educativo y social y una definición precisa para identificar sus componentes. El uso de autoinformes y heteroinformes es la forma preferente de evaluación, pero existen otras. La ubicación de las relaciones de maltrato en los espacios virtuales hace necesario explorar esos escenarios para medir sus peculiaridades, el impacto emocional que ocasionan y los valores que acaban poniendo en juego. La evaluación necesita ponderar el peso de los componentes que interactúan en las dinámicas individuales y grupales, que hacen surgir, mantener y/o cambiar o eliminar las conductas de acoso. Venimos reclamando un giro en ese sentido para mejorar la intervención. La funcionalidad de la evaluación del bullying radica en la identificación de las raíces que lo fundamentan y lo motivan, no solo en la explicación externa o contextual de la frecuencia e el modo como ocurre. Así no erraremos el objeto educativo de la intervención.

PALABRAS-CLAVE BULLYING • EVALUACIÓN DE LA CONDUCTA • CYBERBULLYING • INSTRUMENTOS DE EVALUACIÓN. 


\title{
RESUMO
}

O bullying é um fenômeno que tem despertado interesse educacional e social e uma definição precisa para identificar seus componentes. 0 uso de autoinformes e heteroinformes é a forma predominante de avaliação, mas existem outras. A constatação das relações de maustratos nos espaços virtuais torna necessária a exploração desses cenários para medir suas peculiaridades, o impacto emocional que ocasionam e os valores que acabam colocando em jogo. A avaliação precisa levar em consideração o peso dos componentes que interagem nas dinâmicas individuais e grupais e que fazem surgir, manter e/ou mudar ou eliminar as condutas de assédio. Temos exigido uma mudança nesse sentido para methorar a intervenção. A funcionalidade da avaliação do bullying consiste não só na explicação externa ou contextual da frequência e do modo como acontece, mas também na identificação das raízes que o fundamentam e o motivam. Assim, não erraremos o objeto educativo da intervenção.

PALAVRAS-CHAVE BULLYING • AVALIAÇÃO DO COMPORTAMENTO • CYBERBULLYING • INSTRUMENTOS DE AVALIAÇÃO.

\begin{abstract}
Bullying is a phenomenon that has sparked social and educational interest as well as a precise definition to identify its components. The use of autoinforms and heteroinforms is the main assessment procedure, although others exist. Reports of maltreatment in virtual spaces make it necessary to explore those sceneries so as to measure its idiosyncrasies, the emotional impact they cause, and the values they jeopardize. The assessment must take into consideration the weight of the components that interact in the individual and group dynamics, which give rise to, maintain and/or change or eliminate harassment behaviors. We have demanded change in order to improve the intervention. The effectiveness of bullying assessment consists not only of the external or contextual explanation of how and when it takes place, but also of the identification of the roots on which it is based and is motivated by. Therefore, we will not miss the educational object of the intervention.
\end{abstract}

KEYWORDS BULLYING • ASSESSMENT BEHAVIOR • CYBERBULLYING • ASSESSMENT TOOLS. 


\section{INTRODUCCIÓN}

El objetivo de la evaluación del bullying (AVILÉS MARTÍNEZ, 2002, 2006a, 2013b; OLWEUS, 1998; ORTEGA, 1992) tiene que ver con la recopilación de la información acerca del maltrato en el medio en que se produce. Esto se hace, por una parte, para establecer un diagnóstico que nos ayude a conocer qué está sucediendo en calidad y en cantidad y, por otra, para orientar la intervención más idónea, que a su vez será evaluada a través de los cambios en las dinámicas de los procesos en los que están involucrados los sujetos participantes en el bullying y/o el cyberbullying.

En este artículo, analizamos diversas formas de obtener información sobre el bullying y el cyberbullying y haremos mención especial a las elaboradas y utilizadas por el autor.

Hay muchas razones para interesarse por evaluar las dimensiones o componentes del fenómeno de la intimidación entre iguales. Sin embargo, aquí queremos recalcar aquellas que tienen que ver con motivos pedagógicos y de orientación de la intervención, dado el carácter educativo de esta publicación. 
Por una parte, se puede llegar a tener una idea bastante confusa sobre lo que es el bullying en la comunidad educativa y sobre su extensión en un centro o grupo y a quién/es afecta.

- En primer lugar, conviene reivindicar y establecer un consenso sobre lo que es bullying, lo que puede llegar a serlo $y$ lo que no lo es, en el grupo que consideremos, familias, alumnado o profesorado. En este caso, es crucial acercarse a la naturaleza de sus componentes para discriminar (AVILÉS MARTÍNEZ; ALONSO NATIVIDAD, 2008).

- En segundo lugar, es necesario determinar su extensión. Tan negativo es calcularlo por intuición y decir que "a mí me parece que no existe" o "hay muchísimo" o "eso no pasa”, como vivir preocupado o sensibilizado por la presión de un hecho cercano conocido y terminar viendo bullying detrás de cada incidente. Emplear instrumentos precisos, válidos y fiables será clave (ARORA, 1989; AVILÉS MARTÍNEZ, 2010a; AVILÉS MARTÍNEZ; ELICES, 2007; CEREZO, 2000; FERNÁNDEZ; ORTEGA, 1998; OLWEUS, 1989; ORTEGA; MORA-MERCHÁN; MORA, 1995; RIGBY; SLEE, 1993).

- En tercer lugar, la evaluación puede facilitar el acceso a información, que de otra forma quedaría oculta. No cabe duda de que el silencio es el mayor aliado de los agresores/as y la mayor dificultad para que las fuentes de donde obtener información más cercana hablen y relaten lo que sucede de forma veraz. Además, conviene que la información que podamos obtener, provenga de fuentes diversas y cuanto más diferentes sean, más se complementará el resultado que obtengamos.

Por otra parte, en cada centro suceden cosas diferentes y con prevalencia diversa. Tenemos constancia de ello en investigaciones diversas. (AVILÉS MARTÍNEZ, 2002; DÍAZ-AGUADO, 2008; FUNK, 1997; MARTÍN; MARTIINEZ; DÍAZ-AGUADO, 2010; ROLAND, 2005; SMITH, 2005). Por tanto, la variable centro tomada globalmente parece decisiva. Cada Comunidad Educativa posee factores protectores y de riesgo que hacen variar, por ejemplo, la tipología de la intimidación (AVILÉS MARTÍNEZ; MASCARENHAS, 2007) o los datos de prevalencia en el tiempo de un mismo centro (OLWEUS, 1998). 
Como, en general, el bullying aparece en todos los centros, conviene conocer cuánto bullying sucede y de qué tipo es éste en momentos determinados, para valorar las oscilaciones del mismo en el tiempo y poder así dirigir la intervención.

Además, está justificado evaluar el bullying, por las diferentes preconcepciones que tiene la comunidad educativa acerca de las situaciones de violencia y acoso. Con excesiva frecuencia se confunde el bullying con otras conductas de violencia o con simple conflictividad escolar. Actuar contra el bullying supone emplear métodos específicos de intervención. Por eso, conviene identificar las conductas bullying y separarlas de la conflictividad escolar u otras formas de violencia que exigen tratamientos diferentes.

Conviene conocer la situación real de bullying en el centro educativo para determinar la línea base (evaluación inicial del bullying) y a partir de ella planificar una intervención que nos permita saber si estamos mejorando y obteniendo logros en nuestro propósito. Estos datos servirán para evaluar la efectividad de las estrategias (LONDON, 1994, p.30), así como la necesidad o no de adaptar sus intervenciones.

Finalmente, saber sobre la situación de bullying en el contexto educativo, obtener datos rigurosos de la evaluación, nos legitima para proponer las actuaciones y nos permite canalizar y reunir lo que "conoce" cada sector de la Comunidad Educativa sobre el bullying en su entorno más cercano, permitiendo así desarrollar un proyecto real, el Proyecto Antibullying, herramienta preferente y compromiso real de lucha contra el maltrato en las escuelas. (AVILÉS MARTÍNEZ, 2005, 2013b).

\section{ESTADO DE LA CUESTIÓN}

En estas dos últimas décadas se han utilizado diversas formas para establecer la evaluación del fenómeno del acoso escolar. Aquí haremos mención de algunas de las más significativas y frecuentes y marcaremos las luces y sombras que tiene cada una y que en la práctica, se han manifestado en la consideración y el tratamiento del acoso.

De forma esquemática señalamos a continuación las principales fuentes de donde se ha intentado obtener 
información sobre el bullying y las representamos en lo Quadro 1 (AVILÉS MARTÍNEZ, 2006a):

a) De agresores/as y víctimas. De quienes sufren o provocan los hechos. Estos sujetos aportan información propia sobre cómo se ven y cómo ven los hechos: a través de autoinformes, por ejemplo, cuando contestan sobre lo que les pasa en un cuestionario, a través de entrevistas, cuando comentan historias personales o dibujan experiencias vividas o, incluso, cuando autorregistran sus propias conductas.

b) También podemos obtener una visión más objetiva y complementaria de lo que sucede preguntando a quienes no participan directa y personalmente en los hechos, pero habitualmente los presencian, formando parte de ellos como testigos o, incluso, participando en otros diferentes eventos. Estos métodos nos conducen al uso de heteroinformes, entre los que se han reivindicado como más efectivos los procedimientos sociométricos extensivos a todo el grupo de iguales. En ellos, cada miembro del grupo se pronuncia sobre la participación del resto en la dinámica bullying. Igualmente, la opinión de los adultos que conviven con los chicos/as puede ser requerida como otra fuente de información interesante a tener en cuenta, que toma cuerpo, por ejemplo, en escalas de apreciación, diarios de incidencias o en entrevistas a familias.

c) También podemos obtener información recogiendo directamente la conducta manifestada ante nosotros a través de la observación. Los patios de recreo, las clases, el comedor, el transporte escolar, la calle, etc., y la existencia de personas entrenadas en la observación, nos aportarán datos fiables y objetivos sobre el terreno, de determinados rituales típicos del bullying, de las conductas de los sujetos, así como de las características de las acciones. Se trata de información no sólo cuantitativa, sino fundamentalmente cualitativa.

d) Por último, las redes de apoyo en la escuela, en el ejercicio intencional de su cometido, suponen 
elementos preventivos y también de evaluación del clima escolar y de situaciones de bullying y/o cyberbullying, aunque respeten principios como la confidencialidad entre sus valores inherentes.

A partir del esquema citado en lo Quadro 1, indicamos la gama de métodos de evaluación para obtener información de la conducta específica de bullying:

QUADRO 1 - Posibles fuentes y métodos de obtención de la información en bullying y cyberbullying

\begin{tabular}{|c|c|c|}
\hline AUTOINFORMACIÓN & HETEROINFORMACIÓN & OBSERVACIÓN DIRECTA \\
\hline \multirow{2}{*}{$\begin{array}{l}\text { Entrevistas } \\
\text { Autoinformes } \\
\text { estructurados } \\
\text { Cuestionarios } \\
\text { Autobservación } \\
\text { Autorregistro } \\
\text { Autonarraciones } \\
\text { Dibujos } \\
\text { Mapas de riesgos } \\
\text { Planos del patio } \\
\text { Murales } \\
\text { Pegatinas } \\
\text { Fotografías } \\
\text { Mensajes de texto o } \\
\text { video }\end{array}$} & $\begin{array}{l}\text { Información obtenida de los iguales } \\
\text { Métodos sociométricos: } \\
\text { Nominación de los iguales } \\
\text { Puntuación de los iguales } \\
\text { Dramatizaciones: } \\
\text { Asignación de roles en el bullying } \\
\text { Sistemas de apoyo entre iguales: } \\
\text { Equipos de Acogida } \\
\text { Equipos de Ayuda } \\
\text { Cybermentores }\end{array}$ & \multirow{2}{*}{$\begin{array}{l}\text { Natural } \\
\text { Artificial } \\
\text { Hojas de recuento } \\
\text { Hojas de estimación de frecuencias } \\
\text { Informes técnicos } \\
\text { Alumnado supervisor } \\
\text { Ayudantes de recreo } \\
\text { Equipos de acogida } \\
\text { Equipos de ayuda } \\
\text { Cybermentores } \\
\text { Observación de Role Play } \\
\text { Registro en audio y video } \\
\text { Análisis de sitios en la red: } \\
\text { Mensajes de texto y video } \\
\text { Conversaciones en chats } \\
\text { Interacción en redes sociales } \\
\text { Blogs } \\
\text { Páginas web }\end{array}$} \\
\hline & $\begin{array}{l}\text { Información obtenida de los adultos } \\
\text { Escalas de apreciación o valoración } \\
\text { Diarios de incidencias antibullying } \\
\text { Informes de profesorado anterior } \\
\text { Entrevistas } \\
\text { Hojas de observación } \\
\text { Reuniones de valoración del equipo docente } \\
\text { Nominación del alumnado por parte de adultos } \\
\text { Informes de las familias }\end{array}$ & \\
\hline
\end{tabular}

Fuente: Elaboración del autor.

\section{LA INFORMACIÓN QUE APORTAN LOS SUJETOS IMPLICADOS}

La información que nos proporciona el sujeto en situaciones de bullying o de cyberbullying es tan subjetiva como fundamental. Desde muchos puntos de vista, tener en cuenta la opinión de los sujetos participantes, desde su propia visión, es definitiva a la hora de valorar la toma de decisiones de intervención. También es necesario ponderar los condicionantes que pueden sesgar la información que nos proporcionen.

Desde el punto de vista de la información que aporta quien sufre el bullying, señalamos algunas indicaciones que nos parecen importantes:

- Es necesario considerar el punto de vista de los sujetos que sufren el bullying para determinar la gravedad de las situaciones. Es oportuno considerar el 
umbral de dolor de la víctima, cómo es de dañino un ataque para ella, para hacerse una idea de la magnitud del daño y, por tanto, modular los juicios externos que podamos hacer sobre la relevancia de la agresión. Agresiones que desde fuera nos pueden parecer poco fuertes, de baja intensidad o valoradas como menores, si preguntamos y conocemos lo que significan para la víctima y su dignidad en el contexto en que se producen, podremos valorarlas mejor en su significación adecuada.

- Es necesario considerar también la situación de los sujetos que no tienen conciencia de que son víctimas dentro de su grupo de iguales y que, por tanto, no proporcionan información "veraz" y consciente de su situación de victimización.

- Mucho más generalizada es la situación de las víctimas que no proporcionan información de su situación o niegan, por vergüenza, miedo o intimidación, cuando les intentamos preguntar a través de diversos métodos.

- El punto de vista del sujeto que sufre una situación de victimización también debe ser considerado o tenida en cuenta su opinión, cuando establezcamos las estrategias de actuación ante los hechos. Se ha mostrado muy conveniente para, incluso, no empeorar las situaciones del bullying, que siempre contemos con lo que nos dice el sujeto que está sufriendo el maltrato. Con buena intención, los adultos/as que pretendemos actuar ante una situación, una vez desvelado el maltrato, debemos contar con la opinión de la víctima (AVILÉS MARTÍNEZ; IRURTIA; ALONSO, 2009). Es la víctima quien mejor sabe en qué situación se encuentra. Cualquier información proporcionada por el propio sujeto debe ser considerada importante para guiar la actuación de los adultos/as. Por esta razón, porque la víctima no controla las consecuencias de la información que proporciona a los adultos/as ni las reacciones de estos, es por lo que muchos chicos/as que sufren bullying no lo cuentan ni siquiera a su propia familia. 
Desde el punto de vista de la información que puede aportar quien ejerce la intimidación conviene puntualizar, también, alguna indicación:

- La información aportada por quien ejerce la agresión puede verse condicionada por una actitud bastante frecuente en los agresores/as como es la exculpación (OLWEUS, 1998). Sobre todo cuando son conscientes de sus actos o son descubiertos y "acusados" de ellos, suelen reaccionar exculpándose o ampliando su culpabilidad a la red de agresores/as. Esto minimiza la individualización de la culpa (AVILÉS MARTÍNEZ, 2012). No son pocos los agresores que plantean como atribución causal de lo que hacen, la provocación de los otros, que ellos no fueron los primeros o que a ellos les sucede también.

- Existen también agresores/as en quienes se produce la no conciencia de que sus actos afectan a los otros y a sus sentimientos y emociones, en definitiva, a su bienestar en el grupo. La información que facilitan no está ajustada ya que no son conscientes.

- Otros agresores cuando informan lo que hacen ven sesgada su información por la justificación o la racionalización que atribuyen a lo que hacen (AVILÉS MARTÍNEZ, 2006b), normalmente en grupo y por el proceso grupal en que se produce, la pérdida de valor de la víctima, sus características, el gregarismo, etc.

La información que aporta quien contempla la intimidación puede también verse condicionada por:

- Las presiones de quienes ejercen el poder abusivo pueden hacer callar a los testigos de las agresiones por temor y por falta de apoyo.

- Los prejuicios grupales y la cultura imperante en el grupo sobre ser "chivato" o "delator" frente a una falta de cultura de derechos democráticos, independencia y de ayuda.

- Los déficits en el desarrollo moral de los sujetos y el grupo ante lo justo y lo injusto. 
Sea en entrevistas, mediante cuestionarios o a partir de dibujos, narraciones o figuras y símbolos, la información que nos proporcionan los sujetos inmersos en la dinámica del bullying es fundamental y debe valorarse teniendo en cuenta todas las claves y con todas las precauciones, y, además, debe servir para orientar la intervención educativa.

A continuación destacamos algunos de los métodos más significativos que utilizamos para realizar la evaluación de situaciones de acoso y ciberacoso.

\section{AUTOINFORMES Y HETEROINFORMES}

Una de las formas más usuales que venimos usando para valorar la información que nos proporcionan los sujetos inmersos en situaciones de bullying y/o de cyberbullying son los autoinformes y los heteroinformes. En los primeros son los sujetos directamente implicados quienes aportan su visión, y en los segundos son los sujetos y adultos que contemplan o convienen con el grupo quienes se manifiestan. En general, los cuestionarios sobre el maltrato entre iguales tratan de clarificar ese concepto a las personas que los cumplimentan haciendo hincapié en sus características principales (OLWEUS, 1998). En sus ítems se suelen abordar diferentes aspectos del maltrato como su incidencia, sus formas, la victimización, la intimidación, la contemplación, las atribuciones causales desde cada posición, los sentimientos y emociones de los implicados, etc. (AVILÉS MARTÍNEZ; ELICES, 2007; ORTEGA; MORA-MERCHÁN; MORA, 1995).

Una dificultad reconocida para comparar la incidencia en las distintas investigaciones ha sido no disponer de una misma escala de medida. Los términos utilizados para cuantificar la frecuencia de ocurrencia del bullying han variado desde establecerla con términos vagos como "frecuentemente" hasta concreciones como "más de una vez a la semana" (OLWEUS, 1989). En cualquier caso, es necesaria una diferenciación entre la ocurrencia ocasional de agresiones, que no supone repetición y persistencia, y el bullying sistemático y establecido (AVILÉS MARTÍNEZ, 2002). En nuestro caso, hemos tomado como referencia el criterio de las investigaciones de los profesores Smith en Inglaterra y de la profesora Ortega en España, y 
separamos claramente lo que consideramos un maltrato ocasional y esporádico de lo que es un maltrato sistemático. Para que el bullying sea sistemático consideramos que tiene que tener una recurrencia de al menos una vez a la semana. Por otra parte, en nuestros instrumentos establecemos un tiempo de recuerdo básico para solicitarles esos juicios a los sujetos. El plazo entre dos y tres meses de recuerdo es el establecido para solicitar una valoración a los sujetos sobre la recurrencia y persistencia de la conducta a evaluar. Con ello, aseguramos los componentes de repetición y de duración y estabilidad en el tiempo como dimensiones reconocidas en el fenómeno del bullying (AVILÉS MARTÍNEZ, 2013a; BESAG, 1989; OLWEUS, 1999).

Por otra parte, otra dificultad reconocida a los cuestionarios referidos al acoso radica en la naturaleza del contenido que constituye los propios ítems respecto al constructo en cuestión. En los caso del bullying y el cyberbullying nos parece significativo y central que los instrumentos midan componentes básicos y complementarios que se ponen en juego en las dinámicas de acoso desde cualquiera de los perfiles o contextos, recurrencia, intencionalidad, desequilibrio de poder, focalización, indefensión, exculpación, gratuidad, etc. En el cyberbullying además, existe ausencia de claves socioemocionales, incapacidad de control sobre la situación, inseguridad extendida, imprevisibilidad, percepción de anonimato, audiencia agrandada, permanencia escénica de la agresión, etc. (AVILÉS MARTÍNEZ, 2013d, en prensa). Se medirá acoso si realmente consideramos las dimensiones inherentes al fenómeno y no sólo aspectos externos y situacionales. Y desde todos los perfiles y perspectivas para aportar mayor objetividad.

Los autoinformes más significativos que han venido usándose en la evaluación del bullying han explorado a través de ítems diversas dimensiones del maltrato. Unos de forma más estructurada y fija y otros de forma flexible como son las hojas de chequeo. Indicamos a continuación algunos de ellos para un análisis de forma más pormenorizado.

- Una lista de chequeo es un autoinforme de conductas operativizadas y modificables, tanto positivas como negativas, que chequea la situación respecto al bullying del grupo a explorar. Con ella se obtiene un índice de bullying y otro de agresión a partir de 
determinados ítems, índices sobre los que se puede valorar su evolución con los sucesivos y periódicos pases de la lista. Una de las más conocida es la lista de chequeo My life in school (ARORA, 1989).

- Los autoinformes son cuestionarios que indagan los aspectos más relevantes del bullying o del cyberbullying vistos desde el punto de vista del sujeto implicado. Uno de los más representativos es el Olweus Bully/Victim Questionnaire (OLWEUS, 1989) que ha servido de ejemplo a otros que en lengua castellana se han elaborado después comoelCuestionariosobreintimidaciónymaltratoentreiguales de Ortega, Mora y Mora-Merchán (1995) o el Cuestionario sobre abusos entre compañeros de Fernández y Ortega (1998). También están inspirados en el cuestionario del autor nórdico los autoinformes Preconcimei (AVILÉS MARTÍNEZ, 2000) que analizan el fenómeno del bullying de forma abreviada en tres formas, una para el alumnado, otra para el profesorado y otra para las familias. Recientemente se han publicado en Brasil en lengua portuguesa (AVILÉS MARTÍNEZ, 2013b). La aplicación de autoinformes y heteroinformes conjuntamente y relacionados en el mismo instrumento sucede en un instrumento como el Insebull (AVILÉS MARTÍNEZ; ELICES, 2007). En este instrumento se triangula la información que proporcionan tanto los sujetos implicados en situaciones de bullying como la que dan quienes las contemplan. Con su aplicación se pueden valorar las situaciones en los grupos de convivencia desde esa triple perspectiva. Otros autoinformes significativos que merecen mención son el Peer relations questionnaire - PRQ - (RIGBY; SLEE, 1993) y el Peer relations assessment questionnaires - PRAQ-(RIGBY, 1997) en lengua inglesa y el Cuestionario BULL (CEREZO, 2000) también en castellano.

- Los heteroinformes facilitan información de lo que sucede en las situaciones de acoso desde una posición de mayor objetividad e independencia. Desde la escuela, al valorar las situaciones de acoso y establecer su intensidad y ocurrencia real, los iguales del grupo de convivencia, si son tomados en su totalidad y no 
sólo parcialmente, ofrecen una imagen bastante aproximada de lo que ocurre en el ecosistema escolar (BRONFRENBRENER, 1987; BRONFRENBRENER; MORRIS, 1997). Aunque hay instrumentos que utilizan esta perspectiva (CEREZO, 2000), haciendo hincapié en el componente sociométrico, no lo ponderan en su totalidad como sí lo hacen otros (AVILÉS MARTÍNEZ; ELICES, 2003, 2007). Incluso, hacen participar en esa ponderación no sólo a los pares sino a todos los adultos que conviven con el grupo clase, sean docentes o no, cerrando así la triangulación de la evaluación.

Un ejemplo de heteroinforme que explora la opinión del conjunto del grupo de convivencia es un subtest, Sociobull (AVILÉS MARTÍNEZ;ELICES, 2003), dentro del instrumento Insebull (AVILÉS MARTÍNEZ; ELICES, 2007). Este subtest hace participar a la totalidad del alumnado en la nominación de los iguales partiendo de la tesis de que en el contexto en que sucede el bullying todos los sujetos tienen responsabilidad por acción o por omisión. En este sentido, evalúa las actitudes, los apoyos, las defensas, las decantaciones morales, las oposiciones, los escraches, etc. En definitiva, el posicionamiento moral de cada miembro del grupo ante lo que sucede visto desde un punto de vista individual y sin la presión grupal pública al emitirse anónima y/o confidencialmente.

Análisis del Insebull (AVILÉS MARTÍNEZ; ELICES, 2007), Instrumentos para la Evaluación del Bullying. Se trata de un instrumento que integra un autoinforme (ORTEGA; AVILÉS MARTÍNEZ, 2005) y un heteroinforme (AVILÉS MARTÍNEZ; ELICES, 2003) complementarios y relacionados en los componentes del bullying que explora. $\mathrm{Su}$ análisis factorial identificó factores relevantes en el constructo maltrato entre iguales y estableció correlación entre ellos en cada una de las dimensiones de los cuestionarios que componen el instrumento. La composición de sus ítems va dirigida a la identificación de los componentes relevantes de situaciones de bullying: el desequilibrio de poder, la reiteración de las acciones, la permanencia en el tiempo, la focalización de las agresiones, la conciencia que los sujetos tienen de los hechos y su capacidad de reacción, el desarrollo 
de su red social, su posicionamiento moral, las atribuciones causales que hacen o su estatus grupal. El heteroinforme incorpora además, esas dimensiones vistas desde los iguales, la posición sociométrica de los miembros del grupo respecto al maltrato y las actitudes que adoptan ante el acoso y ante los implicados.

Autoinforme y heteroinforme representan individual y grupalmente mediante gráficos la posición de cada sujeto en las dimensiones del maltrato y en una escala total de previsión de maltrato. Además, permiten comparar la opinión que los adultos y los sujetos tienen sobre lo que pasa. Como puede verse en las Figuras 1, 2 y 3, escolarmente el instrumento aporta una fotografía real de lo que sucede en el grupo-clase, que sirve a los profesionales educativos para tener datos que orientan su intervención.

FIGURA 1 - Gráfico del autoinforme que refleja la situación de cada alumno/a en las variables intimidación y victimización

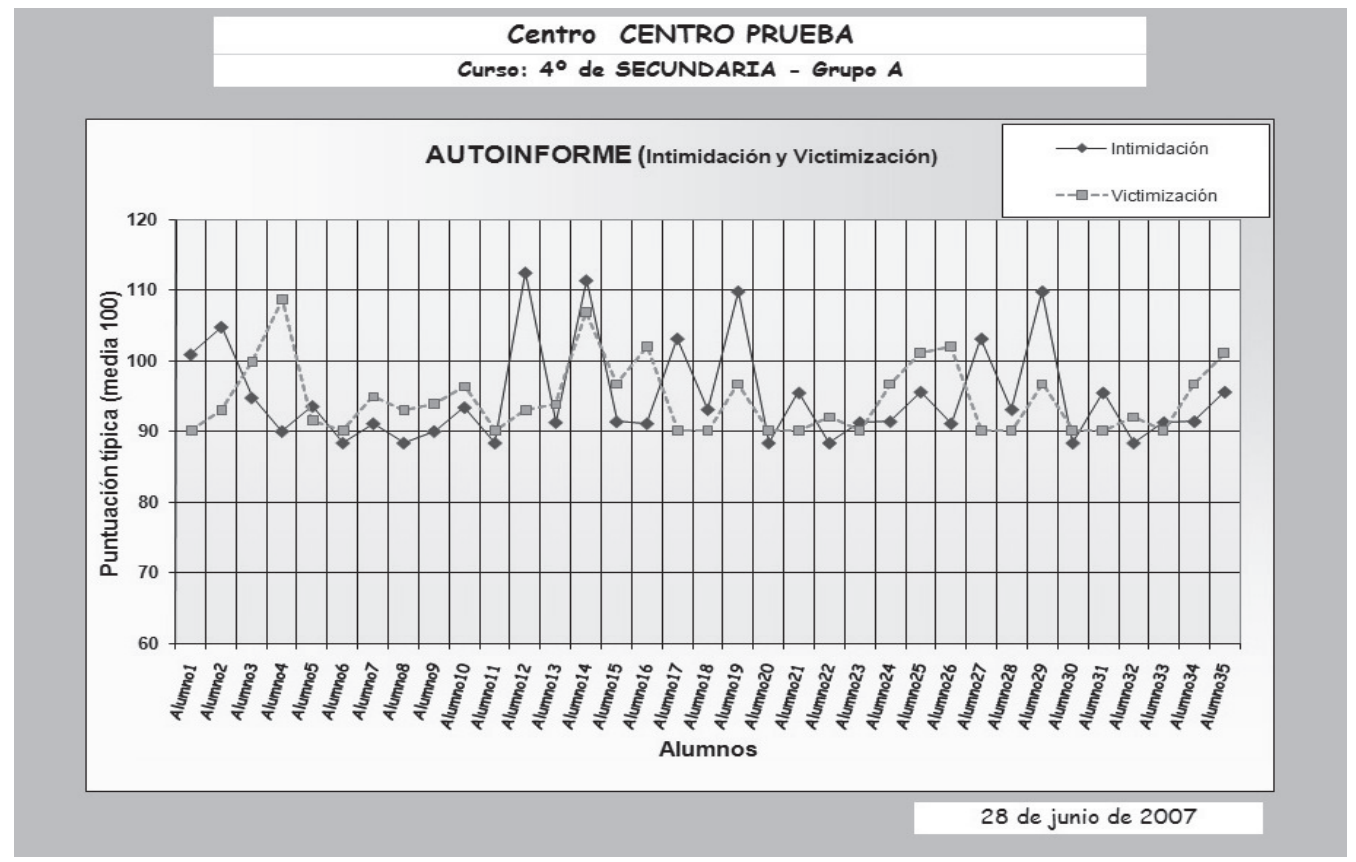

Fuente: Elaboración del autor. 
FIGURA 2 - Gráfico del heteroinforme que refleja la opinión del grupo sobre todos sus miembros en los tres perfiles en el bullying

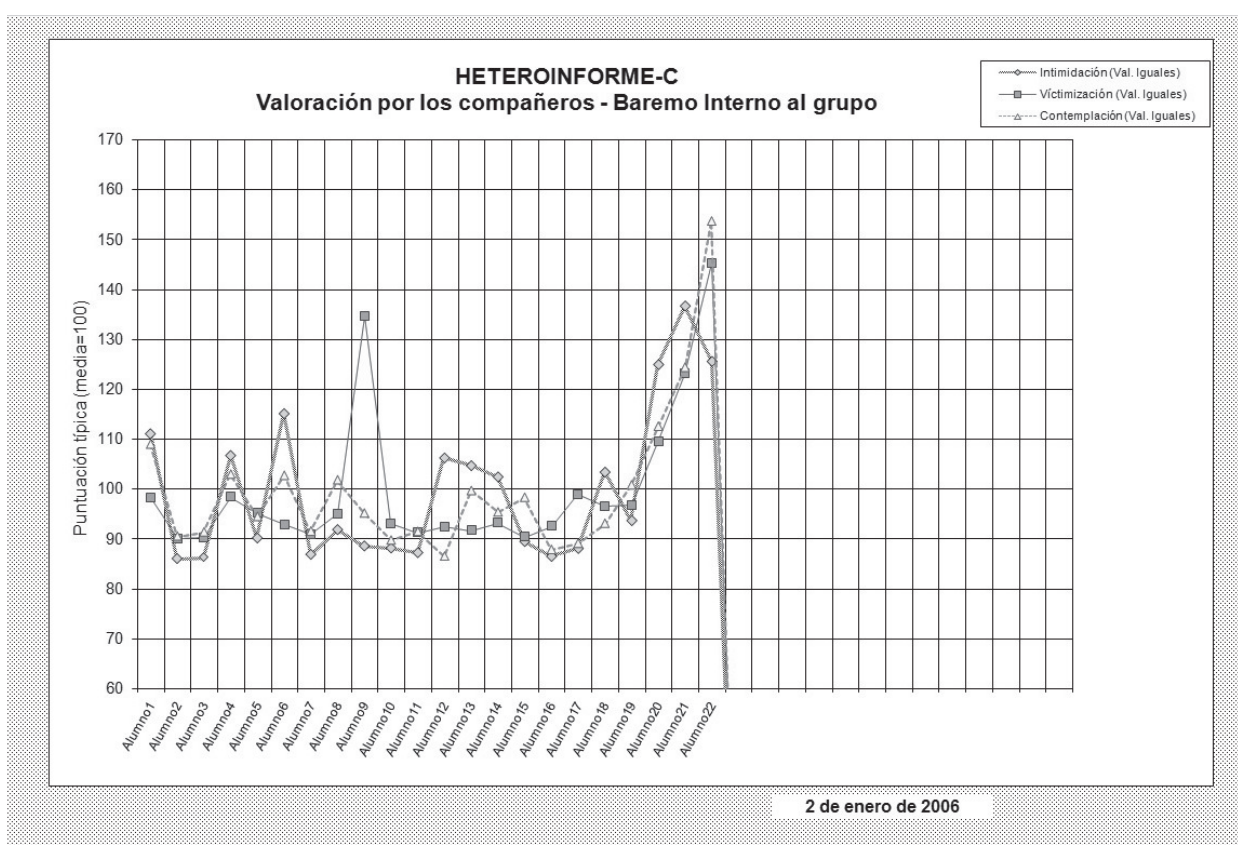

Fuente: Elaboración del autor.

FIGURA 3 - Gráfico que compara la opinión del grupo de iguales y el equipo de profesores para cada sujeto en la variable victimización

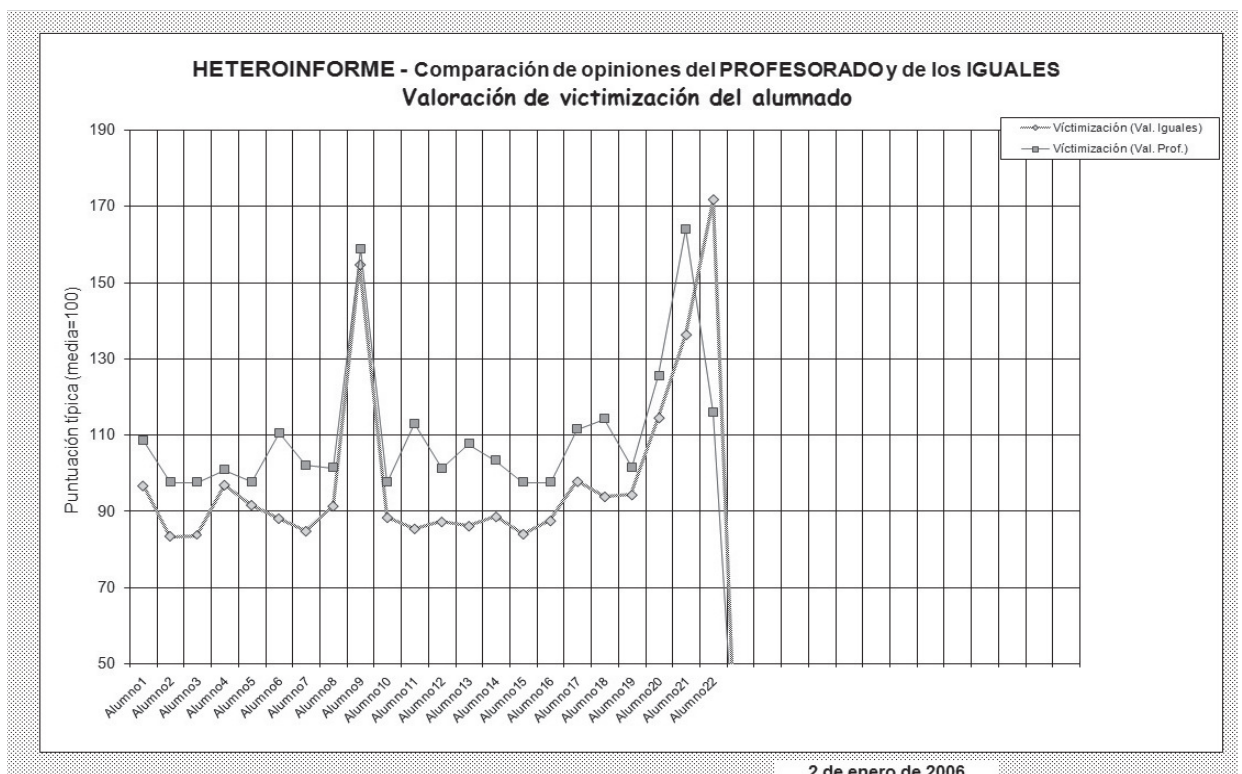

Fuente: Elaboración del autor. 


\section{EL CASO DEL CYBERBULLYING}

A las clásicas formas de maltrato a través de las acciones verbales, físicas o sociales, en los últimos tiempos ha irrumpido con fuerza un maltrato desde los entornos virtuales y los dispositivos móviles, llamado cyberbullying (AVILÉS MARTÍNEZ, 2009, 2010a, 2013b; SHARIFF, 2008; SMITH, 2010), que aporta características específicas, añadiendo al maltrato matices agravantes que lo hacen especialmente dañino y peligroso, tanto para las víctimas, obviamente, como para los agresores/as y espectadores/as.

El caso del ciberbullying ha tenido un menor desarrollo en cuanto a instrumentos. Merece la pena señalar algunos significativos en lengua castellana.

- Cuestionario Ciberbullying (ORTEGA; CALMAESTRA; MORA-MERCHÁN, 2008). Explora las conductas de los adolescentes en el uso de los teléfonos móviles y de internet en relación con el ciberacoso.

- Cybullquest (AVILÉS MARTÍNEZ, 2010b). Ofrece tres formas, para el alumnado, profesorado y familias. En la de los chicos/ as se exploran hábitos online, uso de móviles e internet, prevalencia, tipología y atribución causal del ciberbullying, comunicación de los hechos y el afrontamiento y las salidas que buscan. En la del profesorado, se trabaja con sus preconcepciones y en la gestión que hacen de esas situaciones, y las familias informan del conocimiento, acompañamiento y supervisión que tienen y hacen sobre sus hijos/as, así como de la colaboración que establecen con el centro escolar en la prevención e intervención de estas conductas.

- Cuestionario sobre violencia social online (VELÁZQUEZ, 2010). Es un cuestionario previo a las entrevistas con los sujetos implicados. Adaptado a la situación mejicana, plantea cuestiones de uso de móviles e internet y ocho preguntas abiertas que pretenden servir de guía para esas entrevistas individualizadas.

Como en el bullying, otros instrumentos también se han desarrollado online para la elaboración de diversas investigaciones, pero por una limitación de espacio no los mencionamos aquí. 


\section{TÉCNICAS NARRATIVAS Y/O GRÁFICAS}

Otra forma de obtener información de los propios sujetos es a través de sus trabajos gráficos y/o literarios: un dibujo, una historia, un video, un texto escrito o una narración oral referida al maltrato entre iguales, complementado por la intervención exploratoria del educador/a sobre la producción del alumno/a. Estas técnicas de evaluación cualitativa suponen no sólo un aporte metodológico para acercarse y profundizar en el acoso sino una oportunidad para valorar las intenciones, actitudes, emociones y posicionamientos morales que adoptan los sujetos ante las situaciones de acoso. Desde la Teoría de la Mente (CRICK; DODGE, 1996; MENESINI, 1999; SMORTI, 1999; SUTTON; SMITH; SWETTENHAM, 1999), suele considerarse para explorar y valorar el conocimiento que tienen los sujetos de los procesos del bullying, su cognición social (SMORTI, 1999), y cómo los representan y organizan mentalmente, evaluando el significado que dan a las relaciones negativas del bullying. Así, los elementos narrativos facilitan a los sujetos la tarea que se les propone y que puede ser:

Representar o dibujar una situación, un lugar o un plano del sitio o de los hechos.

- Explicar o decir qué sugiere el dibujo.

- Atribuir significado a una intención o a una situación social.

- Terminar y proponer desenlaces de una secuencia de historias gráficas o escritas.

- Explicar y valorar la conducta de los personajes de una historia escrita, gráfica o visual que se les propone.

Contar por qué hacen eso los personajes, qué sentimientos y emociones les producen, qué posicionamiento moral adoptan en la historia o hecho, qué estrategias de salida proponen a la situación, etc.

Existen diversas vías narrativas y/o gráficas para obtener la información sobre el bullying. Una forma espontánea $y$ directa, cuando el sujeto hace un dibujo o un texto escrito, redacción, carta, etc. En este caso expresa hechos 
directamente, inventados o reales, cercanos a la realidad o proyectados en una historia ficticia. Ejemplo de ello son los textos autobiográficos u originales escritos por chicos/ as que cuentan una historia de maltrato directamente o la expresan de forma delegada en personajes y sujetos inventados u ocultos. Otras veces la narración y el discurso explicativo se producen por el uso de un elemento sugerente o sugerido, que incita a hablar, compartir y debatir. Es una forma más planificada, dirigida, mediada, distante, menos comprometida y más delegada. Si queremos, más 'segura' para los sujetos vulnerables y/o con miedo o vergüenza (SMORTI; CIUCCI, 2000). Ejemplo de estas formas de abordaje las podemos encontrar en herramientas metodológicas de evaluación del bullying como el análisis de casos (AVILÉS MARTÍNEZ, 2004), el uso de fotografías, las tarjetas dibujadas que secuencian una historia (DEL BARRIO et al., 2003), el video (CALLEJÓN et al., 1998) o el uso de cuentos (ALONSO NATIVIDAD, 2013; ALONSO NATIVIDAD; AVILÉS MARTÍNEZ, 2009, 2013). En todos los casos el objetivo del adulto que interviene es conocer las representaciones que se producen en los sujetos, la explicación que dan a los hechos, las emociones que les sugieren, las posturas morales que adoptan y valorar las salidas que plantean para orientar la intervención educativa.

Como el resto de las herramientas de evaluación, el uso de técnicas narrativas presenta ventajas y algún inconveniente. En el haber indicamos la cercanía en las historias contadas, su posibilidad para hacer aflorar situaciones reales y cercanas a los sujetos, la capacidad para expresar las representaciones de los individuos en esas situaciones que, en definitiva, les impulsarán a actuar en un sentido o en otro, la expresión de la cognición social sobre lo que pasa y la valoración de posibles estereotipos y preconcepciones sobre el maltrato, y también, la posibilidad de explorar el fenómeno en niveles de análisis diferentes, el informativo, el comportamental, el emocional y/o el moral. Por otra parte, entre las dificultades que encontramos están el gasto de tiempo para su abordaje individualizado, la posible falta de sinceridad o mediatización social cuando su empleo se haga a nivel grupal, la falta de anonimato que puede verse compensada por la distancia 
de la historia cuando los hechos no son personales y reales, el nivel de competencia lingüística escrita exigida a los sujetos (SMORTI, 1999) o lo restringido de la validez cuando el instrumento se conforme como cerrado y formal (DEL BARRIO et al., 2003), dados los inconvenientes de inferencia de conclusiones, generalización a otras situaciones y las dificultades de identificación personal de los sujetos actores en los actos de maltrato.

En este último sentido, se ha conformado formalmente algún instrumento que explora el bullying desde técnicas narrativas manejando información emocional y de percepción cognitiva de las situaciones de maltrato. El más significativo en español es el SCAN-Bullying (DEL BARRIO et al., 2003). A partir de dibujos de situaciones de bullying trata de explorar en el sujeto lo que le sugieren, que proponga desenlaces a esas situaciones y explique y valore la conducta de los personajes. Ofrece una versión masculina y otra femenina en las historias que presenta.

\section{EL USO DE LAS REDES DE APOYO EN LA ESCUELA}

Sin duda, una herramienta más novedosa, entre las que vamos a analizar aquí, y que venimos utilizando como instrumento de valoración de situaciones de bullying y/o cyberbullying, es el uso de estructuras estables en la escuela, integradas por alumnado formado para intervenir en situaciones de conflicto y/o acoso.

La finalidad de estas redes en la escuela (AVILÉS MARTÍNEZ; IRURTIA; ALONSO, 2009) es fundamentalmente preventiva y de intervención en casos de conflicto y/o bullying, sin embargo también cumplen una función decisiva como herramientas de detección de casos de bullying y de valoración de su evolución en el contexto del grupo aula.

Estas redes pueden estar formadas por alumnado, que periódicamente es supervisado por adultos, perteneciente a estructuras como Equipos de Acogida (AVILÉS MARTÍNEZ, 2012, 2013b), Equipos de Ayuda (AVILÉS MARTÍNEZ; TORRES; VIÁN, 2008), Mediadores Escolares (BOQUÉ, 2005) o e-support (COWIE, 2009) o cybermentores (AVILÉS MARTÍNEZ, 2013a). Estos alumnos, en unos modelos son elegidos por los propios 
pares y en otros por el profesorado. En cada caso reciben una formación específica y tienen un cometido definido por la naturaleza de cada modelo. Sin embargo, son estructuras que aportan, unas más que otras, determinados elementos que ayudan en los procesos de evaluación del bullying y el cyberbullying. Como son:

- La detección de situaciones de acoso presencial o en la red.

- La evolución de los casos ya detectados tras la gestión e intervención en los mismos.

- Datos cualitativos sobre la valoración de necesidades y/o intereses de las partes.

- Datos de cumplimiento de compromisos a que llegan los sujetos implicados.

- Datos sobre el afrontamiento de las situaciones que hacen los sujetos.

- Datos sobre su posición moral ante las salidas propuestas en la práctica restaurativa.

- Datos sobre las posturas frente a la norma o frente al régimen disciplinario escolar.

La aportación que hacen a la evaluación estas redes de apoyo en la escuela en ningún caso tienen un componte delator o acusador. Aunque en la naturaleza de algunos de los modelos se incorporan señas de identidad como la confidencialidad o la preservación de identidades de los beneficiarios de la red, eso no es óbice para que cumplan las funciones indicadas anteriormente. En otros modelos, sin embargo, se manejan identidades directas de los sujetos, por lo que los aportes a la evaluación son nominales e identificados.

La ventaja más evidente de una herramienta como los iguales para la evaluación de situaciones de bullying y/o cyberbullying es, por una parte la inmediatez que da la propia convivencia en el seno del grupo donde surgen los problemas, y por otra, la presencia con capacidad de percepción y registro en los tiempos y espacios en que esas situaciones acaecen. Abordar a tiempo los casos es, en sí mismo, una garantía de prevención y supone contar con la información precisa desde el primer instante para poder intervenir. 
También por su propia naturaleza se trata de un método de evaluación eminentemente cualitativo que aporta mucha información de calidad y situacional de los casos y que puede ser usado en todos los momentos del proceso de los casos de bullying, en la detección, durante la gestión y evolución del caso y en el desenlace y toma de decisiones para buscar una salida.

Por otra parte, el hecho de que el alumnado democráticamente, en algún caso como en los Equipos de Ayuda, deposite su confianza en determinados alumnos/as de la clase para hacer ese trabajo, confiere a las aportaciones que hacen en los momentos de evaluación de los casos componentes de autenticidad, sinceridad y legitimidad que ayudan más a comprometer a las partes en la resolución y en la búsqueda de salidas. Así lo atestiguan ciertos datos de la evaluación de alguno de los modelos que hemos llevado a cabo (AVILÉS MARTÍNEZ, 2013c; AVILÉS MARTÍNEZ; TORRES; VIÁN, 2008) y que respaldan otras en otros países (ELLIS; MARSH; CRAVEN, 2009; NAYLOR; COWIE, 1999; NAYLOR et al., 2009).

\section{PERSPECTIVAS}

El uso de instrumentos formales en los procesos de evaluación del bullying en los contextos escolares exige incorporar a ellos dimensiones que no solo los hagan prácticos para las tareas de evaluación, sino más funcionales y complementarios para tareas preventivas y de intervención sobre el acoso.

Esas dimensiones pasan, por ejemplo, por hacer en ellos cada vez más intencionales y visibles los rasgos o componentes característicos del acoso (AVILÉS MARTÍNEZ; ALONSO NATIVIDAD, 2008). Tanto desde un punto de vista general como en cada uno de los perfiles del bullying y del cyberbullying, se dan componentes que tienen que ser, primero objeto de medida y después tarea de intervención, si lo que queremos es valorar e incidir sobre lo que realmente constituye el acoso y no sobre aspectos externos, anecdóticos y/o coyunturales. Así, respecto a las dimensiones básicas, la evaluación ha de fijar su mirada sobre componentes como la intencionalidad, la recurrencia y el desequilibrio de poder (AVILÉS MARTÍNEZ, 2006a, 2013a; 
BESAG, 1989; OLWEUS, 1998; RIGBY, 1996) que cursan con situaciones de bullying y/o cyberbullying. Al mirar a la víctima hemos de considerar su indefensión propia y en el grupo, su miedo o vergüenza a hablar de lo que le sucede o su red social que marcará o no algunas vías de salida. En definitiva, su vulnerabilidad personal y social. El agresor/a nos debe aportar componentes de planificación, focalización, sentimientos de superioridad, inconsistencia de argumentación o exculpación de sus acciones. Los testigos nos hablan de componentes como la inhibición grupal para actuar ante los hechos, el silencio sobre los hechos que manifiestan con los adultos o el conocimiento cómplice que tienen. En el caso del cyberbullying (AVILÉS MARTÍNEZ, 2013a), la evaluación ha de fijarse especialmente en los desequilibrios respecto al poder, la seguridad y la capacidad de control sobre la situación que se establecen entre los implicados.

En relación con la propuesta anterior, también es necesario incorporar en los instrumentos de evaluación de forma intencional y planificada el conjunto de contenidos que se activan en cualquier relación interpersonal y que ponen en juego determinados valores en los contextos en que sucede el bullying o el cyberbullying: las preconcepciones que los sujetos tienen sobre la violencia y el ejercicio de poder abusivo, las actitudes ante las situaciones de maltrato, los sentimientos $y$ emociones que les generan, la postura moral que les merece y/o las acciones intencionales que les incitan a tomar.

No en vano, cuando preguntamos a los diferentes miembros de la comunidad educativa, encontramos distintas posturas sobre lo que se considera abuso, lo que podría ser maltrato y/o lo que no lo es claramente. En ocasiones, lo que el profesorado considera inadmisible, por ejemplo, en las formas de una relación interpersonal, su propio alumnado lo ve como normalizado e irrelevante. No lo valora, ni lo considera. Se hace necesario tener presentes las preconcepciones que tienen los actores que interactúan sobre el bullying y/o el cyberbullying, no solo para entender lo que sucede ajustadamente, sino para enfocar las posibles salidas o planificar la intervención. Sucede de forma similar con las actitudes de los personajes involucrados en los casos. 
Conocer a través de la evaluación una actitud determinada en un sujeto ante una propuesta de salida a un caso, puede modular la presión que los adultos hagan con la norma o con el régimen disciplinario de la escuela sobre él con el fin de conseguir una salida digna para todos, incluido el agresor/a. Esto es especialmente importante al llevar a cabo la práctica restaurativa (AVILÉS MARTÍNEZ, 2012, 2013b; COWIE; JENNIFER, 2007) o la puesta en práctica del Método Pikas (PIKAS, 1989) o el Método de No-Inculpación (ROBINSON; MAINES, 2003). La actitud del profesorado o de las familias ante los hechos, por ejemplo, puede informar sobre la trivialización social, la resistencia ante el abuso o sobre la implicación prevista en la resolución de los casos.

La información que nos da la evaluación sobre los sentimientos y las emociones que provocan en los sujetos estas situaciones ayuda a valorar el umbral del dolor o las dimensiones del daño (víctima); la consciencia de los hechos, el pensamiento consecuencial o la patologización de la conducta (agresores/as); la conexión moral o la capacidad empática (agresores/as y espectadores/as); el grado de interiorización social que el maltrato puede tener en un sector determinado (comunidad educativa); etc.

Sin duda, la evaluación de la historia escolar del maltrato entre iguales en el seno de una comunidad educativa, es decir, qué situaciones han sucedido, qué actitudes hemos adoptado y qué hemos hecho hasta ahora para resolverlas, también nos aportará un glosario de preconcepciones, argumentaciones, iniciativas, justificaciones, exculpaciones, etc., que en definitiva nos hablará del posicionamiento moral explícito e implícito que individual y colectivamente la comunidad ha ido construyendo ante los hechos. Posicionamiento que, sin duda, podemos deconstruir a partir de la reflexión y toma de decisiones individuales y colectivamente. Esa reflexión que nos aportan los datos de la evaluación histórica del maltrato entre iguales en nuestro contexto nos ayudará, sin duda, también a pensar y adoptar acciones nuevas y concretas que de forma consciente y colectiva nos impulsarán a luchar contra el abuso.

Preconcepciones, actitudes, sentimientos y emociones, postura moral y acciones intencionales son aspectos 
relevantes, todos ellos ámbitos que mirar para una evaluación inicial del contexto escolar, cuando una comunidad educativa se propone dar pasos encaminados a construir herramientas estables para combatir el bullying, como es la elaboración del Proyecto Antibullying de la comunidad educativa (AVILÉS MARTÍNEZ, 2005).

Finalmente, es necesario intensificar en las dinámicas de evaluación del bullying y el cyberbullying la participación de las redes de iguales de las que hemos hablado más arriba. Desde una perspectiva cualitativa, aportan la información inmediata y directa de lo que sucede en los grupos, sirven de avanzadilla como medida preventiva no sólo de los conflictos, sino también de las situaciones de bullying y/o cyberbullying y son un canal directo de valoración de la efectividad y consistencia de las intervenciones para erradicar el acoso. Especialmente idóneo es contar con ellas en los contextos virtuales y en las redes sociales para la prevención del cyberbullying. Pueden aportar modulación, contrapeso y corrección de situaciones de comunicación y presión grupal, y además valores diferentes a los que en un momento determinado estén vigentes en el grupo. Además, pueden constituirse de forma estructurada y reconocida en el centro educativo como redes de ayuda, e-support (COWIE, 2009) o como cybermentores (AVILÉS MARTÍNEZ, 2013a). También pueden difundir prácticas saludables en Internet $\mathrm{y}$ en las redes sociales interviniendo en temas como la gestión de la imagen digital, la privacidad de la imagen y los datos personales, la comunicación interpersonal virtual o riesgos como el sexting, cyberbullying o grooming. Su papel puede ser importante tanto en los momentos iniciales de la evaluación, al detectar las situaciones, pero sobre todo, en momentos en que sea necesario una valoración de la efectividad de las medidas tomadas o como garantes de que los individuos inmersos en una situación de cyberbullying respetan los compromisos a que han llegado en la resolución de un caso. 


\section{CONCLUSIONES}

Hemos querido destacar en este artículo la complejidad de la evaluación en las situaciones de bullying y de cyberbullying. Es necesario diversificar las fuentes de obtención de datos, triangulando, en la medida de lo posible, la información que tienen los sujetos directamente implicados con la que aportan tanto los que no lo están y aparecen como testigos, como con la opinión de los adultos/as que conviven con ellos.

Por otra parte, se hace necesario fijar la mirada de quienes participan en la evaluación más sobre los procesos, y en lo que hay detrás de ellos, que sobre los productos finales. Los datos son importantes y en el caso del bullying y el cyberbullying mucho más, pero es imprescindible identificar en los procesos evaluadores, los componentes relevantes del acoso, los argumentos que manejan los alumnos/as, las motivaciones que mueven a los sujetos a participar y/o posicionarse en uno y otro sentido, los sentimientos y la gestión de las emociones que hacen los implicados y la postura moral por la que se decantan los no implicados que son inmensa mayoría. Estos datos nos situarán la diana de la intervención nítidamente, permitiendo, en el marco de una educación en valores, poder intervenir educativa e intencionalmente en una suerte de programa de desarrollo moral, en el que a través del currículo de la escuela, podamos trabajar los valores que se ponen en juego en las dinámicas de abuso de poder con intervenciones específicas en el aula, y podamos ayudar al alumnado en su período formativo a construir relaciones afectivas, sociales y morales justas, equilibradas y saludables.

Sin duda, hemos resaltado que la evaluación y sus instrumentos no pueden quedarse en los momentos de detección y cuantificación de datos, sino que deben ir más allá. En el caso del bullying y el cyberbullying, deben ayudar a regular el desarrollo de la gestión de los casos y la efectividad de las medidas adoptadas para resolverlos. Debemos incorporar la evaluación sobre el propio Proyecto Antibullying como herramienta de supervisión y progreso.

Por otro lado, el caso del cyberbullying exige a la evaluación, por una parte un aumento de los focos de obtención de la información, dado el espacio en que se produce, incorporando a 
perfiles entre los iguales como agentes de evaluación en el seno de los grupos, y por otra, redoblar la necesidad de evaluación sobre la toma de decisiones morales y el ejercicio de valores de los sujetos en los espacios virtuales, donde no hay otros ojos con autoridad moral que los propios y los de los iguales de la red. Por eso, la evaluación debe situarse en esos parámetros si queremos medir la coherencia de los sujetos entre lo que dicen y hacen cuando no hay agentes de autoridad mirando o su autenticidad, honestidad y sinceridad cuando están de por medio en la red componentes de ilusión de anonimato, posible suplantación de identidad o un contexto de desinhibición. En este sentido, el compromiso individual y colectivo y la observancia de un código común de buenas prácticas en la red serán decisivos.

En fin, en una tarea común como tiene que ser la elaboración del Proyecto Antibullying en la comunidad educativa, la evaluación tiene que estar incorporada de forma global sobre el propio proyecto, pero también de manera específica en varias de sus fases iniciales, especialmente cuando nos dispongamos a analizar la realidad del contexto en que se quiere llevar a cabo el proyecto y cuando determinemos la situación real del alumnado respecto al bullying y/o al cyberbullying. Utilizar herramientas adecuadas e idóneas a la realidad educativa concreta será tarea de los profesionales educativos que trabajen en ella, pero ha de hacerse con la mayor participación posible de los sectores que componen la comunidad educativa y utilizando todas las fuentes y perspectivas posibles que consigan arrojar luz sobre un fenómeno multicausado como es el maltrato entre iguales.

\section{REFERENCIAS}

ALONSO NATIVIDAD. La utilización de la literatura infantil para la convivencia. In: UNIVERSIDADE ESTADUAL DE CAMPINAS. Curso de formación, II, GEPEM. Campinas: Unicamp, 2013.

ALONSO NATIVIDAD; AVILÉS MARTÍNEZ, J. M. Análisis del bullying a través de adaptación de un cuento infantil tradicional. Propuestas de trabajo en el aula. Amazônica: Revista de Psicopedagogia, Psicologia Escolar e Educação, v.2, n. 1, p. 76-96, 2009. 
La prevención del abuso a través del trabajo de los cuentos tradicionales. In: CONGRESO INTERNACIONAL GALAICO-PORTUGUÉS DE PSICOPEDAGOGÍA, 22., 2013, Braga. Anais... Braga, 2013. p. 11-13.

ARORA, C. M. J. Bullying: action and intervention. Pastoral Care, p. 44-47, set. 1989.

AVILÉS MARTÍNEZ, J. M. Cuestionario PRECONCIMEI para la evaluación del maltrato entre iguales. Valladolid: Autor, 2000.

La intimidación entre iguales (bullying) en la Educación Secundaria Obligatoria. Validación del Cuestionario Cimpi y estudio de incidencia. 2002. Tese (Doctoral) - Universidad de Valladolid, 2002.

¿Cómo abordar un caso de bullying? In: Curso de verano. Prevención de riesgos laborales en la enseñanza, 4., Bilbao: Instituto Vasco de Ergonomía, 2004. p. 143-173.

Intervenir contra el bullying en la Comunidad Educativa. 2005. Disponível em: <www.concejoeducativo.org>. Acesso em: 8 dez. 2005.

Bullying: el maltrato entre iguales. Agresores, víctimas y testigos en la escuela. Salamanca: Amarú, 2006a.

Diferencias de atribución causal en el bullying entre sus protagonistas. Electronic Journal of Research in Educational Psychology, v. 4, n. 2, p. $136-148,2006 b$.

Ciberbullying. Diferencias entre el alumnado de secundaria. Boletín de Psicología, n. 96, p. 79-96, 2009.

Éxito escolar y ciberbullying. Boletín de Psicología, n. 98, p. 73-85, 2010a.

Cybullquest. Cuestionario de ciberbullying. Valladolid: Autor, 2010b.

Manual contra el bullying. Guía para el profesorado. Lima: Libro Amigo, 2012.

Análisis psicosocial del cyberbullying. Claves para una educación moral. Papeles del psicólogo, v. 34, n.1, p. 65-73, 2013a.

Bullying. Guia para educadores. São Paulo: Mercado de Letras, 2013b.

Evaluación de la red de Equipos de Ayuda del Instituto de Educación Secundaria Parquesol. Valladolid: Memoria del Departamento de Orientación, 2013c.

Diferencias entre bullying presencial y cyberbullying. In: CONGRESO INTERNACIONAL GALAICO-PORTUGUÉS DE PSICOPEDAGOGÍA, 22., 2013, Anais... Braga, 2013d.

AVILÉS MARTÍNEZ, J. M.; ALONSO NATIVIDAD. Análisis de componentes identificativos de la violencia en el contexto escolar. Violencia, conflicto y maltrato. Itinerario de frontera. In: CONGRESSO NACIONAL DE PSICOLOGÍA DA SAÚDE, 7., 2008, Anais... Porto: ISPA Ediciones, 2008. p. 119 -129.

AVILÉS MARTÍNEZ, J. M.; ELICES, J. A. Sociobull. Procedimiento sociométrico de evaluación del bullying. Valladolid: Autores, 2003.

Insebull: instrumentos para la evaluación del bullying. Madrid: CEPE, 2007. 
AVILÉS MARTÍNEZ, J. M.; IRURTIA, M. J.; ALONSO, M. N. Equipos de ayuda entre el alumnado y satisfacción de la comunidad educativa. Amazônica: revista de psicopedagogia, psicologia escolar e educação, v. 2, n. 1, p. 51-60, 2009.

AVILÉS MARTÍNEZ, J. M.; MASCARENHAS, S. A. Diferencias de percepción en la tipología del maltrato entre iguales (bullying) entre sus participantes a partir de dos muestras de enseñanza secundaria obligatoria de España (Valladolid) y Brasil (Amazonas/ Humaitá). Presentación y Actas del IX Congreso internacional galego-portugués de psicopedagogía, 2007, p. 154-166. A Coruña: Universidad de A Coruña.

AVILÉS MARTÍNEZ, J. M.; TORRES, N.; VIAN, M.V. Equipos de ayuda, maltrato entre iguales y convivencia escolar. Revista de Investigación Psicoeducativa, v. 6 , n. 3, p. 357-376, 2008.

BESAG, V. Bullies and victims in school. Milton Keines: Open University, 1989.

BOQUÉ, M. C. Tiempo de Mediación. Barcelona: CEAC, 2005.

BRONFENBRENNER, U. La ecología del desarrollo humano. Madrid: Paidós, 1987.

BRONFENBRENNER, U.; MORRIS, P. The ecology of developmental process. In: DAMON, W. (Org.). Handbook of child psychology: theoretical models of human development. 5. ed. Nueva York: Wiley, 1997. p. 993-1029.

CALLEJÓN, M. et al. Un día más. Madrid: El defensor del menor en la Comunidad de Madrid, 1998.

CEREZO, F. BULL-S. Test de evaluación de la agresividad entre escolares. Madrid: Grupo Albor-Cohs, 2000.

COWIE, H. Cyberbullying and peer support. Learning Science Forum. Graduate School of Education. Hiroshima: Hiroshima University, 2009.

COWIE, H.; JENNIFER, D. Managing violence in schools. A whole-school approach to best practice. London: PCP, 2007.

CRICK, N.R.; DODGE, K.A. Social information-processing mechanisms in reactive and proactive aggression. Child Development, v. 67, p. 993-1002, 1996.

DEL BARRIO, C. et al. Representaciones acerca del maltrato entre iguales, atribuciones emocionales y percepción de estrategias de cambio a partir de un instrumento narrativo: SCAN-Bullying. Infancia y Aprendizaje, v. 26, n. 1, p. 63-78, 2003.

DÍAZ-AGUADO, M. J. Manual de interpretación de indicadores de la calidad de la convivencia en Educación Secundaria Obligatoria, 2008. Disponible en: <http://www. redconvivencia.net/v2_2/herramienta/Manual.pdf>. Acceso en: 12 dic. 2011.

ELLIS, L.A.; MARSH, H.W.; CRAVEN, R.G. Addressing the challenges faced by early adolescents: a mixed-method evaluation of the benefits of peer support. American Journal of Community Psychology, v. 44, p. 54-75, 2009.

FERNÁNDEZ GARCÍA, I.; ORTEGA, R. Cuestionario sobre abusos entre compañeros. In: FERNÁNDEZ, I. (Ed.) Prevención de la violencia y resolución de conflictos. El clima escolar como factor de calidad. Madrid: Narcea, 1998. 
FUNK, W. Violencia escolar en Alemania. Revista de Educación, v. 313, p. 53-78, 1997.

LONDON. Department for education. Bullying: don't suffer in silence. An anti-bullying pack for schools. London: DfEE, 1994.

MARTÍN, J.; MARTÍNEZ, A. R.; DÍAZ-AGUADO, M. J. Estudio estatal sobre la convivencia escolar en la educación secundaria obligatoria. 2010. Disponível em: $<$ https://sede.educacion.gob.es/publiventa/detalle.action?cod=13567>. Acesso em: jan. 2011.

MENESINI, E. Bullying and emotions. 1999. Disponível em: <http://www.gold. ac.uk/tmr/reports/aim2_firenze2.html>. Acesso em: fev. 2005.

NAYLOR, P.; COWIE, H. The effectiveness of peer support systems in challenging school bullying: the perspectives and experiences of teachers and pupils. Journal of Adolescence, v. 22, p. 476-479, 1999.

NAYLOR, P. B. et al. The PEPE anti-bullying intervention in schools: cross-national evaluation in England \& Spain. In: SYMPOSIUM "BULLYING, CHILDREN AT RISK, COPING STYLES, AND THE IMPACT OF ANTI-BULLYING INTERVENTIONS IN SCHOOL" AT EUROPEAN SOCIETY FOR DEVELOPMENTAL PSYCHOLOGY, EUROPEAN CONFERENCE ON DEVELOPMENTAL PSYCHOLOGY, 14., 2009. Anais...Vilnius, Lithuania, 2009.

OLWEUS, D. Prevalence and incidence in the study of antisocial behaviour: definition and measurements. In: Klein, M. (Org.): Cross-national research in self-reported crime and delinquency. Dordrecht, The Netherlands: Khiwer, 1989.

Conductas de acoso y amenaza entre escolares. Madrid: Morata, 1998.

OLWEUS, D. Sweden. In: SMITH, P. K. et al. (Org.). The nature of school bullying: a cross-national perspective. London: Routlegde, 1999. p. 7-27.

ORTEGA, R. Violence in schools. Bully-victims problems in Spain. European Conference on Developmental Psycholog, 5., 1992. Sevilla, p. 27.

ORTEGA, R.; AVILÉS MARTÍNEZ, J. M. Autoinforme para la evaluación del bullying. Valladolid: Autores, 2005.

ORTEGA, R.; CALMAESTRA, J.; MORA-MERCHÁN, J. Cyberbullying. Journal of Psychology and Psychological Therapy, v. 8, n. 2, p. 183-192, 2008.

ORTEGA, R.; MORA-MERCHÁN, J. A.; MORA, J. Cuestionario sobre intimidación y maltrato entre iguales. Sevilla: Proyecto Sevilla Anti-Violencia Escolar. Universidad de Sevilla, 1995. Disponível em: <http:/|www.laecovi.es/ documents/Alum_secundaria_maltrato(Ortega_Mora-Merchan_Mora)8p.pdf>.

PIKAS, A. The common concern method for the treatment of mobbing. In: ROLAND, E.; MUNTHE, E. (Org.). Bullying: An international perspective. London: David Fulton, 1989. p. 91-105.

RIGBY, K. Bullying in schools. And what to do about it. Londres: Jessica Kingsley, 1996.

. The peer relations assessment questionnaires (PRAQ). Point Lonsdale,

Victoria, Australia: The Professional Reading Guide, 1997. 
RIGBY, K.; SLEE, P. T. Peer relations questionnaire. Adelaide: University of South Australia, 1993.

ROBINSON, G.; MAINES, B. Crying for help. The no blame approach to bullying. Bristol: Lucky Duck, 2003.

ROLAND, E. La respuesta al bullying en Noruega. In: CONGRESO LA CONVIVENCIA EN LAS AULAS, PROBLEMAS Y SOLUCIONES. Anais... Madrid: Ministerio de Educación y Ciencia, 2005.

SHARIFF, S. Cyber-bullying. New York: Routledge, 2008.

SMITH, P. K. Violencia escolar y acoso: factores de riesgo familiares. In: SANMARTÍN, J. (Org.) Violencia y escuela. Valencia: Centro Reina Sofía para el estudio de la violencia, 2005. p. 59-76.

SMITH, P.K. Cyberbullying: the European perspective. In: MORA-MERCHAN, J.; JÄGER, T. (Ed.). Cyberbullying: A cross-nationalcomparison. Landau: Verlag Emprische Padagogik, 2010.

SMORTI, A. Theory of mind in bullying: a methodological reassessment. 1999. Disponível em: <http://www.gold.ac.uk/tmr/reports/aim2_firenze1.html>. Acesso em: 22 set. 2005.

SMORTI, A.; CIUCCI, E. Narrative strategies in bullies and victims. Aggressive Behaviour, v. 26, p. 33-48, 2000.

SUTTON, J.; SMITH, P.K.; SWETTENHAM, J. Bullying and "theory of mind": a critique of the "social skills deficit" view of anti-social behavior. Social developmental, v. 8, p.117-127, 1999.

VELÁZQUEZ REYES, L. M. Adolescentes en tiempos de oscuridad. La violencia social online en estudiantes de secundaria. Toluca: e.ikon digital, 2010.

JOSÉ MARÍA AVILÉS MARTÍNEZ

Profesor y doctor en Psicología por la Universidad de Valladolid, España. Jefe del Departamento de Orientación del Instituto de Educación Parquesol de Valladolid, España aviles@uva.es 